\title{
Review: primary care counselling improves psychological symptoms in patients with psychological and psychosocial problems
}

\author{
Rowland N, Bower P, Mellor Clark J, et al. Effectiveness and cost effectiveness of counselling in primary care. Cochrane \\ Database Syst Rev 2001;(4):CD001025 (latest version 11 May 2001).

\section{QUESTION: In patients with psychological and psychosocial problems, is counselling in primary care effective and cost effective?}

\section{Data sources}

Studies were identified by searching databases including Medline; EMBASE/Excerpta Medica; PsycLIT; CINAHL; the Cochrane Library; and the Cochrane Collaboration Depression, Anxiety, and Neurosis Register of Randomized Controlled Trials (RCTs) and controlled clinical trials (for trials completed by April 1998). Search terms included primary health care, counselling, psychotherapy, general practice, and clinical psychology. A specialist journal was hand searched, bibliographies of relevant studies were scanned, and experts were contacted.

\section{Study selection}

Published and unpublished studies in all languages were selected if they were RCTs; examined patients with psychological or psychosocial problems who were suitable for counselling; used a clear definition of counselling compatible with that of the British Association of Counselling; used counselling offered by trained practitioners; included outcomes of clinical effectiveness, patient satisfaction, and health service utilisation; and compared counselling with a control treatment. Trials of patients on psychotropic drugs in addition to counselling were included if a direct comparison could be made between treatments. Exclusion criteria were specialist counselling or structured treatments (eg, cognitive behavioural therapy, behavioural therapy, and problem solving therapy).

\section{Data extraction}

Data were extracted on methodological quality, participants, type and duration of interventions, and outcomes and cost effectiveness.

Sources of funding:

University of Leeds and

University of

Manchester.

For correspondence: Ms N Rowland,

Bootham Park

Hospital, York, UK

Nancy.Rowland@

excha.yhs-tr.northy.nhs.uk.

\section{Main results}

4 English language RCTs (678 patients) using up to 12 sessions of face to face counselling of individual patients offered by a range of trained practitioners met the selection criteria. Follow up ranged from 6 weeks to 9 months. Results focused on post-treatment follow up $(6$ to $12 \mathrm{wk})$. Data on psychological symptom levels were pooled from the 4 RCTs; patients receiving counselling had better psychological symptom levels than did those receiving usual care (standardised mean difference $-0.30,95 \%$ CI -0.49 to -0.11 ). 3 RCTs reported generally high patient satisfaction levels with counselling. No clear difference was shown between counselling and usual care for health service utilisation (4 RCTs) or cost effectiveness (1 RCT).

\section{Conclusions}

In patients with psychological and psychosocial problems, counselling in primary care improves psychological symptom levels. Patient satisfaction with counselling seems to be high. Data are lacking on the cost effectiveness of counselling.

\section{COMMENTARY}

Because up to a third of patients in primary care have psychological conditions requiring care, ${ }^{1}$ the review of primary care counselling for psychological and psychosocial problems by Rowland $e t$ al is welcome. The review examined studies of counselling in primary care done by trained counsellors. On the surface, it seems that the use of counsellors in primary care is appropriate; however, there are a few caveats. Because the studies had differing inclusion criteria, data for patients with major depression, dysthymia, or anxiety disorders were pooled together in the analyses. In addition, the type of counselling varied from study to study. The review would have been strengthened if specific psychological symptoms had been analysed separately to determine whether certain ones are more amenable to counselling. The results may not apply to all psychological symptoms. Finally, because counselling was not compared with psychotropic drugs, it is unclear whether 5 minutes of counselling and the additional use of drugs would be as beneficial as the reliance on multiple sessions of counselling.

Even with these cautions, a counsellor in primary care practice seems to be beneficial and should be a service that is covered by health insurance. This review clearly shows that patients prefer counselling.

Philip J Baty, MD Grand Rapids Family Practice Residency Grand Rapids, Michigan, USA

1 Pringle M, Laverty J. A counsellor in every practice? BMJ 1993;306:2-3. 